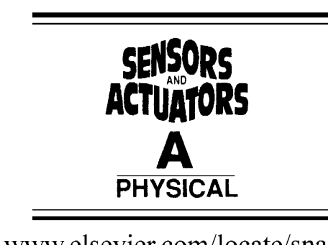

www.elsevier.com/locate/sna

\title{
Novel structure for large area image sensing
}

\author{
M. Fernandes ${ }^{a, *}$, M. Vieira ${ }^{a}$, R. Martins ${ }^{b}$ \\ a ISEL, Electronics and Communications Department, R. Cons. Emídio Navarro, 1949-014 Lisboa, Portugal \\ ${ }^{\mathrm{b}}$ Department of Materials Science, Faculty of Sciences and Technology, New University of Lisbon, \\ Campus da Caparica, 2829-516 Caparica, Portugal
}

Received 22 September 2003; received in revised form 18 March 2004; accepted 25 March 2004

Available online 15 June 2004

\begin{abstract}
This work presents preliminary results in the study of a novel structure for a laser scanned photodiode (LSP) type of image sensor. In order to increase the signal output, a stacked $\mathrm{p}-\mathrm{i}-\mathrm{n}-\mathrm{p}-\mathrm{i}-\mathrm{n}$ structure with an intermediate light-blocking layer is used. The image and the scanning beam are incident through opposite sides of the sensor and their absorption is kept in separate junctions by an intermediate light-blocking layer. As in the usual LSP structure the scanning beam-induced photocurrent is dependent on the local illumination conditions of the image. The main difference between the two structures arises from the fact that in this new structure the image and the scanner have different optical paths leading to an increase in the photocurrent when the scanning beam is incident on a region illuminated on the image side of the sensor, while a decreasing in the photocurrent was observed in the single junction LSP. The results show that the structure can be successfully used as an image sensor even though some optimization is needed to enhance the performance of the device.
\end{abstract}

(C) 2004 Elsevier B.V. All rights reserved.

Keywords: Large area; Image; Photodiode

\section{Introduction}

In recent works the suitability of a-SiC:H structures to be used as large area image sensors was proven [1,2]. The advantages of this approach are quite obvious like the feasibility of large area deposition and on different substrate materials (e.g. glass, polymer foil, etc.), the simplicity of the device and associated electronics. Nevertheless, and due to the physical process involved, charge collection modulation by the local illumination conditions, the device exhibits reduced signal amplitude forcing the use of lock-in amplification. In order to increase the signal level a different structure was investigated, which consists on two stacked $\mathrm{p}-\mathrm{i}-\mathrm{n}$ structures separated by a light-blocking layer. The readout process is similar to the previous one [1] with the difference that in this structure the image and the scanning beam are incident on opposite sides of the sensor. The working principle of the laser scanned photodiode (LSP) sensor is based on the measurement of the modulated photocurrent generated by a small light beam under different local illumination conditions. The technique is similar to the laser-beam induced photocurrent (LBIC) that was used by several groups

\footnotetext{
* Corresponding author.

E-mail address: mfernandes@deetc.isel.ipl.pt (M. Fernandes). URL: http://www.isel.ipl.pt.
}

to determine material properties [3] and characterize solar cells $[4,5]$.

\section{Experimental}

\subsection{Sample preparation}

The proposed structure was fabricated in a three-chamber load-lock UHV-system by plasma enhanced chemical vapour deposition (PE-CVD) at $13.56 \mathrm{MHz}$ radio frequency on a glass substrate.

The structure is composed by two stacked $\mathrm{p}-\mathrm{i}-\mathrm{n}$ diodes separated by a Cr light-screening layer (see Fig. 1). The p-doped layers in both diodes were fabricated introducing methane during the deposition process, in order to decrease the conductivity of the material, which is of crucial importance for device operation. The light-screening layer consists of $290 \mu \mathrm{m} \times 290 \mu \mathrm{m}$ Cr pixels with $40 \mu \mathrm{m}$ spacing and was fabricated by thermally evaporating a $90 \mathrm{~nm} \mathrm{Cr}$ film, followed by photolithography and reactive ion etching. This layer prevents the light crossing one junction from getting to the other one. The patterning is performed to decrease the lateral conductivity, which has a negative effect on the spatial resolution of the device. The electrical contacts are formed by a semitransparent $\mathrm{Cr}$ contact on the top 


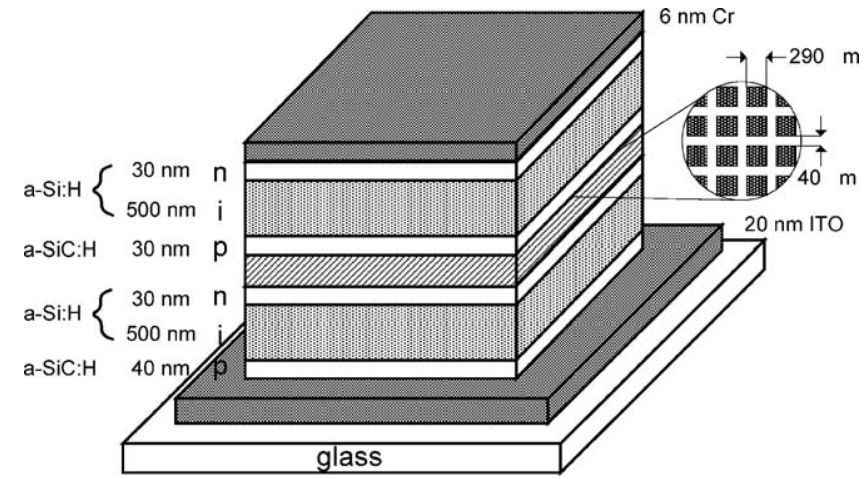

Fig. 1. Structure of the device.

and an ITO contact on the bottom of the structure, as seen in Fig. 1.

\subsection{Readout}

The image readout process relies on the same working principle of the LSP sensor [1] with the difference that the image and the scanning beam are incident on opposite sides of the sample, as shown in Fig. 2. This approach simplifies the optical system since image and scanning beam have different optical paths. The scanning beam is generated by two low power $(<5 \mathrm{~mW})$ solid-state lasers with different different wavelengths $(552$ and $633 \mathrm{~nm})$. The beam deflection is controlled by a two-axis deflection system capable of high-speed scan, where two additional photodiodes provide the synchronization signals for the scanning beam position information necessary for the image restoration process.

The current from the device is amplified by a current to voltage converter with selectable gain and converted to digital format by a signal acquisition card installed on a computer. The data is stored as a matrix of photocurrent values which provide information about local illumination conditions on each position of the active area of the device. Further processing algorithms like fixed pattern noise suppression are performed by software.

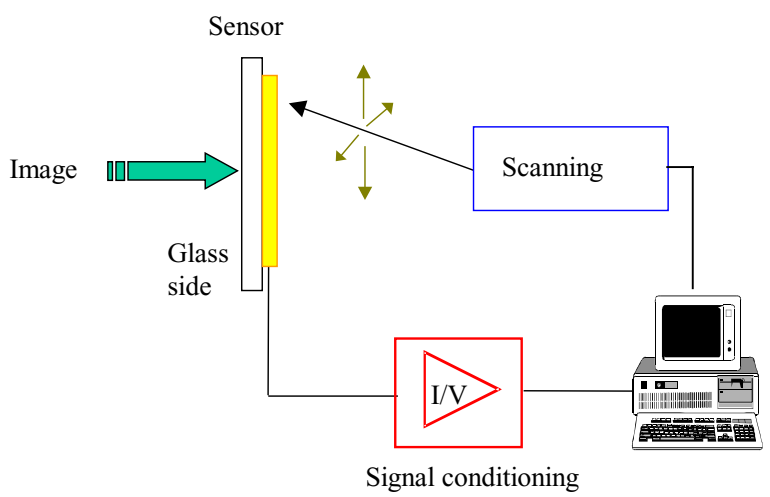

Fig. 2. Sketch of the readout system.

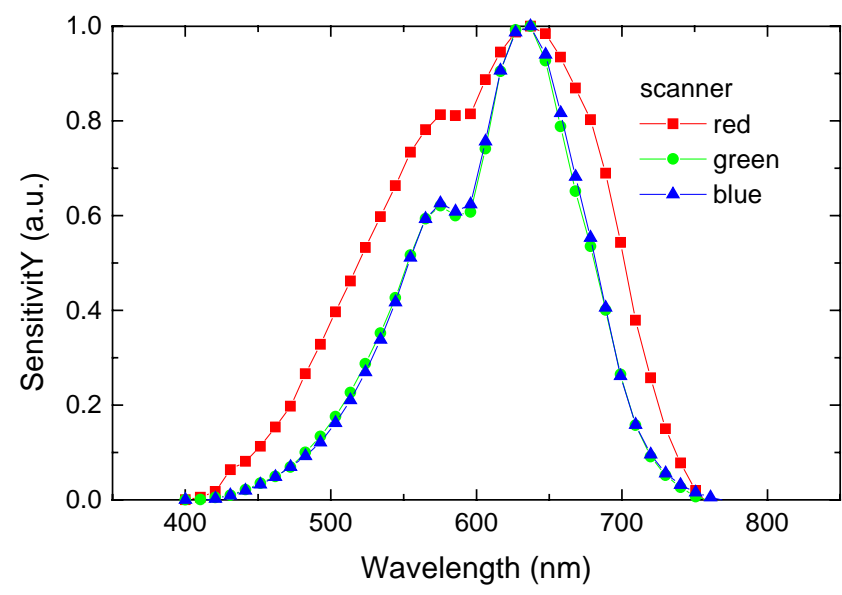

Fig. 3. Plot of the wavelength dependent sensitivity with the scanning beam incident from the ITO side.

\section{Results and discussion}

\subsection{Image responsivity}

In order to evaluate the sensor responsivity to different wavelengths of the scanner and image, the photocurrent generated by the scanning beam was measured with a lock-in amplifier under different illumination conditions. The lock-in amplification provides a simple method of measuring the scanning beam-generated photocurrent under different background illumination conditions. In these measurements three LED's with 450,550 or $650 \mathrm{~nm}$ wavelengths were used as scanning beam source, while the wavelength of the image was changed in the range $400-800 \mathrm{~nm}$.

Fig. 3 presents the normalized sensitivity as function of the wavelength with the scanner incident from the $-\mathrm{p}$ side (ITO side) and the image on the opposite side. In this mode the plot follows the typical spectral response of a $\mathrm{p}-\mathrm{i}-\mathrm{n}$ amorphous silicon photodiode. With blue and green scanning beams the curves are coincident which is the result of these wavelengths being absorbed close to the $\mathrm{p}-\mathrm{i}$ interface.

The increase in the sensitivity observed with the red scanner is a consequence of the almost constant light absorption profile throughout the $\mathrm{p}-\mathrm{i}-\mathrm{n}$ structure, even beyond the light-screening layer since it is not completely opaque and some residual light from the scanning beam can cross it, giving rise to a photocurrent increment.

If the position of scanner and image are interchanged the sensitivity widens since now the absorption of the scanner light occurs strongly close to the $\mathrm{i}-\mathrm{n}$ interface. The green scanner presents the wider sensitivity, since the absorption occurs throughout the top structure while the absorption of blue light is confined to a region closer to the $\mathrm{i}-\mathrm{n}$ interface.

In the high wavelength region of the spectrum the sensitivity decreases abruptly as a consequence of the penetration of some residual light from the image across the light-screening layer. When the number of photons crossing 


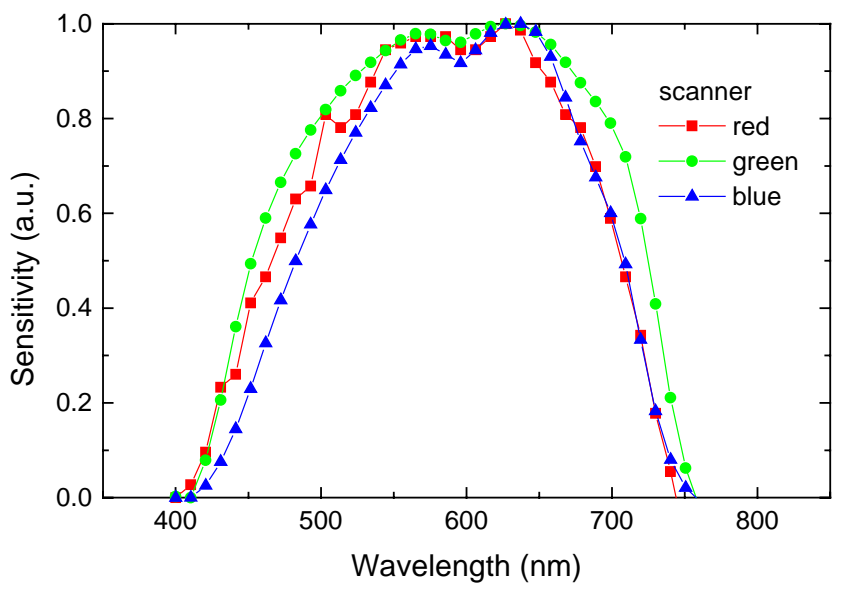

Fig. 4. Plot of the wavelength dependent sensitivity with the scanning beam incident from the $\mathrm{Cr}$ side.

the light-screening layer increase the collection efficiency of the ones coming from the scanner is lowered. This fact is more evident in Fig. 5, which is relative to a similar structure except for the absence of the light-screening layer. In this structure the response in high wavelength region decreases significantly even becoming negative, relative to the dark value [6]. This is a consequence of the decrease in the collection efficiency of the scanner-generated carriers in the first $\mathrm{p}-\mathrm{i}-\mathrm{n}$ diode caused by the absorption of the long wavelength photons from the image throughout the two diodes.

Although, the light-screening layer is not continuous (letting some light pass through) there is no significant degradation of the sensor response in the upper region of the visible spectrum (Fig. 4), when compared with a similar structure without the light-screening layer (Fig. 5).

\subsection{Line scanning}

Fig. 6(a) shows the signal obtained in several fast line scans with different image intensities. In this experiment the central area of the sensor is illuminated while the outer

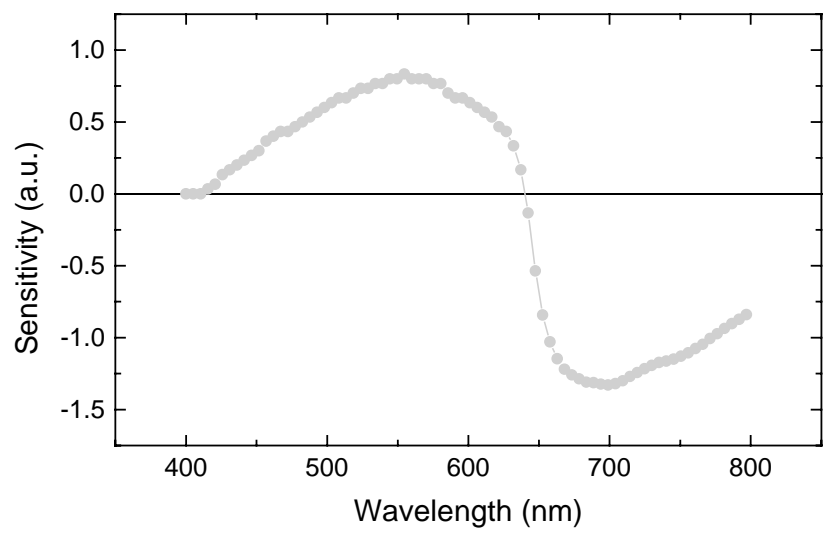

Fig. 5. Plot of the wavelength dependent sensitivity with a green scanner for a structure without a light-screening layer.
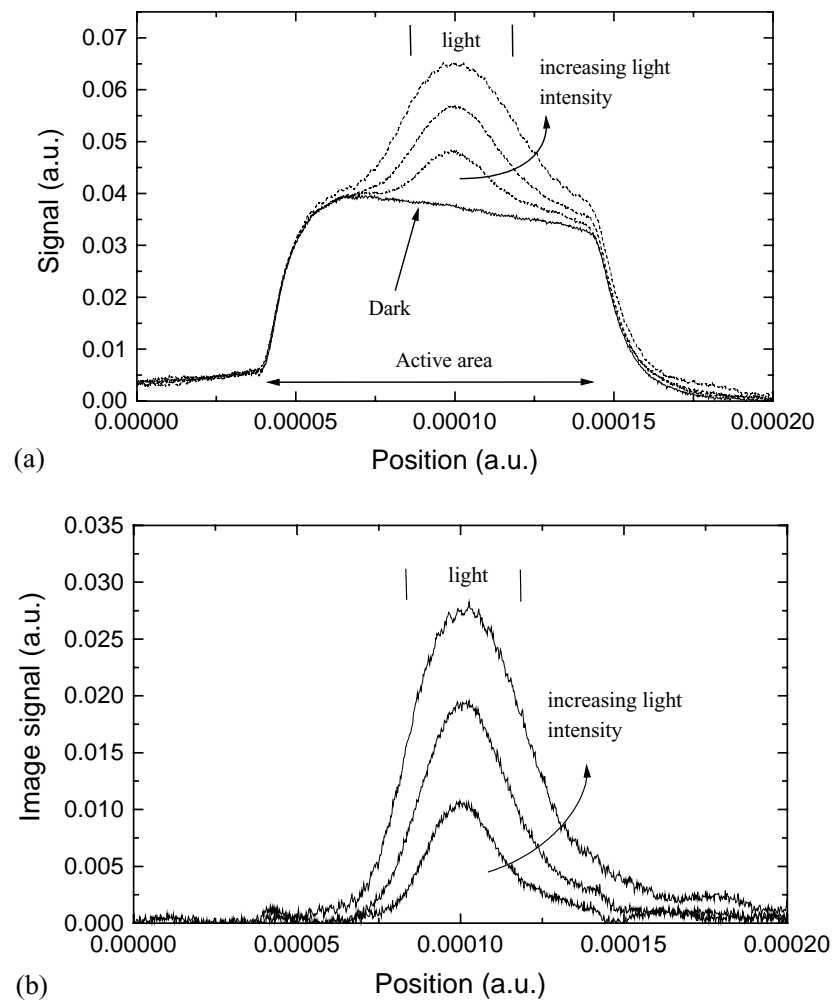

Fig. 6. (a) Signal obtained on several line scans with different image intensities; (b) Image signal obtained by dark signal subtraction.

region is kept in dark. The scan speed is 10,000 lines per second and the sensor active area of $\sim 1 \mathrm{~cm}$.

When the scan is performed with no image (dark) the photocurrent increases when the scanning beam enters the active area of the device with a rise time on the order of $10 \mu \mathrm{s}$ and remains almost constant until the scanning beam exits the sensor, where the same transient is observed. The rise and fall time of the signal are limiting factors for the scan speed and are dependent on the impedance of the signal amplifier. Under these conditions there is no current generation in the first junction (glass side), since the blocking layer prevents the scanner light from reaching it. The fact that the current is not completely constant throughout the sensor can be ascribed to the variation in the $\mathrm{Cr}$ film thickness across the sensor.

When the sensor is illuminated with some light pattern the photocurrent remains the same in dark zones (for low image intensities) and increases (proportionally to the light intensity) in the illuminated zones, while in the single $\mathrm{p}-\mathrm{i}-\mathrm{n}$ structures it decreases [7]. In the last situation the charge generation occurs in both junctions at the same position so the charges are generated across the whole sensor, which justifies the increase in the photocurrent.

With the scanner out of the illuminated region, an increase in the photocurrent is still observed due to lateral currents in the doped layers of the first sensor. This problem can be avoided if the conductivity of the doped layers is decreased by the incorporation of carbon as in the second junction. 


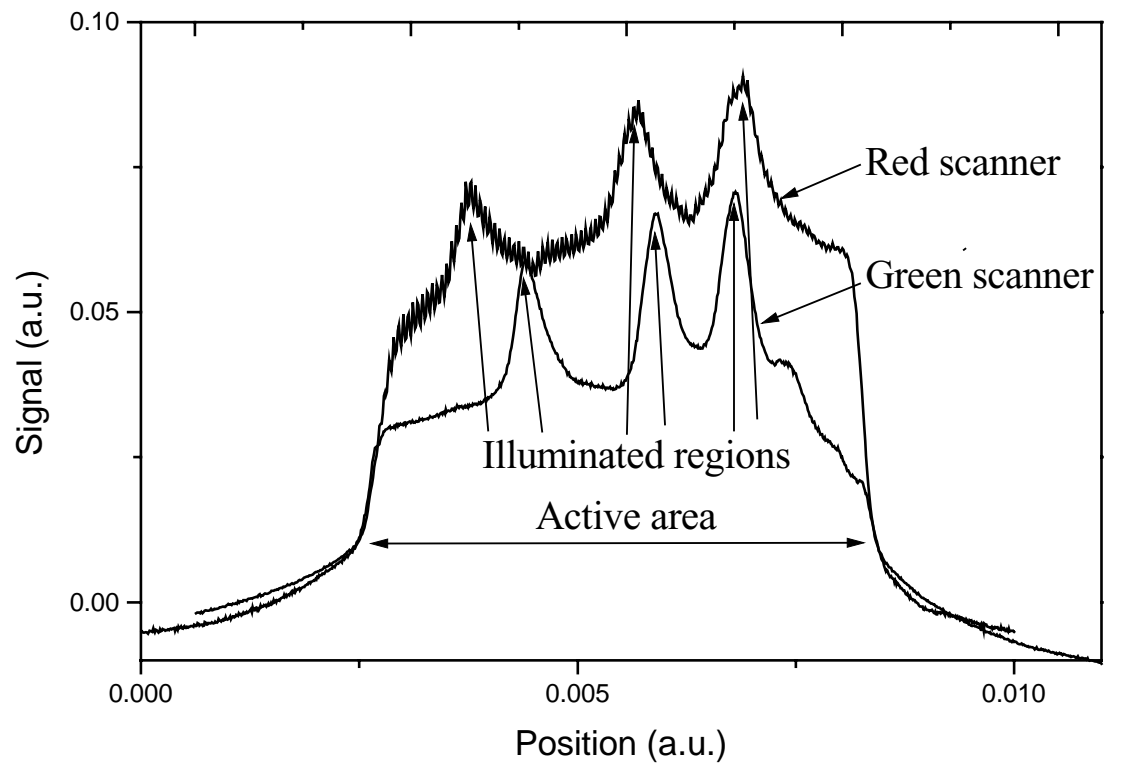

Fig. 7. Signal obtained in two line scans, with a red and a green scanner, on a sensor with three illuminated regions on its active area.

The lateral currents are responsible for a smearing effect on the image, which increases with the light intensity due to the increase of these currents.

The image signal (Fig. 6b) is obtained by the subtraction of the dark scan, which also eliminates the fixed pattern noise due to thickness or material properties variation in the different layers and represents the simple signal processing necessary to restore the image.

As one can state by the analysis of Fig. 7, which represents the signal recorded in one line scanning of a sensor illuminated in three different regions of its active area, the signal is influenced by the scanning beam wavelength, with the green scanner presenting a better spatial resolution as a result of the lower lateral currents and also due to a lower penetration of the light in the structure. An evidence of the non-ideality of the light-screening layer (due to the patterning) can be seen with a red scanner. The scanner light can reach the second junction by the spaces between the $\mathrm{Cr}$ islands and the scanning beam-induced photocurrent increases. The same effect is not observed with green scanning beam since virtually no light can reach the second junction. Another consequence of the penetration of the light from the scanning beam in the second diode is the decrease of the signal difference between the illuminated and the dark zones, thus, degrading de sensor performance.

\section{Conclusions}

This work presents the first results obtained with a novel structure for an LSP type image sensor. The results show that a higher signal can be achieved with this approach, thus simplifying the signal processing electronics, while keeping the same functionality. The data also shows that the spectral response of the sensor does not change significantly with the scanning beam wavelength.

The metal light-screening structure has proven to be effective to optically decouple the two $\mathrm{p}-\mathrm{i}-\mathrm{n}$ structures, while maintaining good electrical conductivity in the transversal direction and reducing the lateral one. Further improvements in the light-screening layer, namely its opacity, and on the composition of the doped layers are needed to optimize the device performance, namely the spatial resolution.

\section{Acknowledgements}

The authors would like to thank Dr. Yuriy Vygranenko for the fabrication of the sensors used in this work.

\section{References}

[1] M. Vieira, M. Fernandes, J. Martins, P. Louro, A. Maçarico, R. Schwarz, M. Schubert, Laser scanned p-i-n photodiode (LSP) for image detection, IEEE Sens. J. 1 (2) (2001) 158-167.

[2] M. Vieira, M. Fernandes, J. Martins, P. Louro, A. Maçarico, R. Schwarz, M. Schubert, Improved resolution in a P-I-N image sensor by changing the structure of the doped layers, Mater. Res. Soc. Symp. Proc. 609 (2000) A14.

[3] J. Marek, Light-beam-induced current characterization of grain boundaries, J. Appl. Phys. 55 (2) (1984) 318-326.

[4] S.A. Galloway, A. Brinkman, K. Durose, P.R. Wilshaw, A.J. Holland, A study of the effects of post-deposition treatments on CdS/CdTe thin film solar cells using high resolution optical beam induced current, Appl. Phys. Lett. 68 (1996) 3725.

[5] J. Cartensen, G. Popkirov, J. Bahr, H. Foll, CELLO: an advanced LBIC measurement for solar cell local characerization, in: Proceedings of the 16th European Photovoltaic Solar Energy Conference, Glasgow, 2000, pp. 1627-1630. 
[6] M. Vieira, P. Louro, M. Fernandes, A. Fantoni, Optical confinement in a double colour laser scanned photodiode (D/CLSP), Sens. Actuators A, 2003.

[7] M. Fernandes, M. Vieira, I. Rodrigues, R. Martins, Large area image sensing structures based on a-SiC:H: a dynamic characterization, Sens. Actuators A, 2003.

\section{Biographies}

Miguel Fernandes was born in Portugal in 1970. In 1993, he became researcher in the Center of Excellence for Microelectronics and Optoelectronics Processes-UNINOVA, Lisbon, Portugal. He graduated in physics and materials engineering by the Faculty of Sciences and Technology from the New University of Lisbon in 1995. Currently, he is Assistant Professor in Electronics Department of ISEL, Lisbon, Portugal and investigator in the Group of Applied Research in Microelectronic Optoelectronic and Sensors-GIAMOS in the same institution.

Manuela Vieira was born in Lisbon, Portugal in 1951. She graduated in physics by the Faculty of Science of the University of Lisbon in 1974. In 1986, she received the Master of Science in solid state physics-microelectronic by the New University of Lisbon. At that time she became Auxiliary Professor of semiconductor and microelectronics in
ISEL, Lisbon where she is now Coordinator Professor in semiconductor. In 1993, she received the $\mathrm{PhD}$ in semiconductor materials from the New University of Lisbon. Currently, she is the head of the Group of Applied Research in Microelectronic Optoelectronic and Sensors-GIAMOS/ISEL in Lisbon, Portugal. Dr. M. Vieira has several scientific papers and more than 12 years of experience in the field of thin films and devices, her research activities have been mainly related to the transient analysis and characterization of the transport properties of the semiconductor materials and device characterization.

Rodrigo Ferrão de Paiva Martins was born in New Lisbon, Angola in 1951. He graduated in electronics engineering by the University of Luanda in 1974. In 1977, he received the MSc in amorphous semiconductors technology by the University of Dundee, Scotland. In 1982, he received the $\mathrm{PhD}$ in energy conversion and semiconducting materials by the New University of Lisbon. In 1986, he became Associated Professor in the Faculty of Sciences and Technology of the New University of Lisbon and received the aggregation by the same institution in 1988 in Semiconductors and Microelectronics. Currently, he is full professor in FCT/UNL and President of the Material Science Department. Dr. R. Martins has several scientific papers, was a member of several scientific comities and coordinator of several European projects over the last years. His main scientific interests are in the field of optoelectronic materials, amorphous semiconductor technology and applications. 